\title{
The Introduction of Capillary Structures in 4D Simulated Vascular Tree for ART 3.5D Algorithm Further Validation
}

\author{
Beatrice Barra*a, Sara El Hadji ${ }^{\mathrm{a}}$, Elena De Momi ${ }^{\mathrm{a}}$, Giancarlo Ferrigno ${ }^{\mathrm{a}}$, Francesco Cardinale ${ }^{\mathrm{b}}$, \\ Giuseppe Baselli ${ }^{\mathrm{a}}$ \\ ${ }^{\text {a} P o l i t e c n i c o ~ d i ~ M i l a n o, ~ E l e c t r o n i c ~ I n f o r m a t i o n ~ a n d ~ B i o e n g i n e e r i n g ~ D e p a r t m e n t, ~ P i a z z a ~ L e o n a r d o ~ d a ~}$

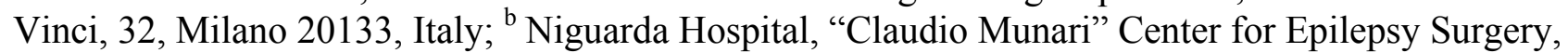 \\ Piazza Ospedale Maggiore 3, Milano 20162, Italy.
}

\begin{abstract}
Several neurosurgical procedures, such as Artero Venous Malformations (AVMs), aneurysm embolizations and StereoElectroEncephaloGraphy (SEEG) require accurate reconstruction of the cerebral vascular tree, as well as the classification of arteries and veins, in order to increase the safety of the intervention. Segmentation of arteries and veins from 4D CT perfusion scans has already been proposed in different studies. Nonetheless, such procedures require long acquisition protocols and the radiation dose given to the patient is not negligible. Hence, space is open to approaches attempting to recover the dynamic information from standard Contrast Enhanced Cone Beam Computed Tomography (CE-CBCT) scans. The algorithm proposed by our team is called ART 3.5 D. It is a novel algorithm based on the postprocessing of both the angiogram and the raw data of a standard Digital Subtraction Angiography from a CBCT (DSA$\mathrm{CBCT}$ ) allowing arteries and veins segmentation and labeling without requiring any additional radiation exposure for the patient and neither lowering the resolution. In addition, while in previous versions of the algorithm just the distinction of arteries and veins was considered, here the capillary phase simulation and identification is introduced, in order to increase further information useful for more precise vasculature segmentation.
\end{abstract}

Keywords: contrast-enhanced cone-beam CT; brain angiography; classification of veins capillary and arteries

\section{INTRODUCTION}

Segmentation and visualization of brain vessels are important in many neurological diagnostic and therapeutic applications. Embolization of cerebral arteriovenous malformations (AVMs), or their radio-surgical treatment, make the three-dimensional (3D) reconstruction of brain vasculature of utmost importance [1,2] and several studies indicate that $3 \mathrm{D}$ visualization of brain vasculature within multimodal imaging proved to be useful in epilepsy surgery, nonetheless still not being used to its full potential in clinical practice [3-7]. Besides the vascular tree reconstruction, the detached visualization of arteries and veins could be advantageous to surgical treatment planning, making procedures easier and safer.

Several techniques are used for the separation of arterial from venous structures, such as Magnetic Resonance Angiography based (MRA) and 4D CT-based techniques [8, 9]. Though being successful, those methods are often difficult to apply in a clinical context, since the acquisition and processing of several images is expensive and time consuming for the surgeons.

We developed a novel algorithm for post-processing the angiogram and the raw data of a standard Digital Subtraction Angiography from a Cone Beam CT (DSA-CBCT) allowing arteries and veins segmentation and labelling based on basis functions. The algorithm takes into account the contrast dynamics, which had been overlooked in the standard arteriography computation. The algebraic problem solved by ART 3.5D is well posed as to the proportion of unknowns of voxel-wise description of contrast dynamics and of projection data. This is made possible modeling the TICs with a basis function model and reconstructing them only for the voxels included in the prior angiographic segmentation. Voxel's TICs reproduce the implicit and usually overlooked dynamic information contained in the CBCT acquired data due to the transit of the contrast bolus. Thought being limited to simulations, ART 3.5D proved to be suitable for arteries and veins distinct segmentation classification and preliminary results proving the suitability of ART 3.5D for arteries and veins classification have already been obtained on simulated datasets comprising just arteries and veins structures

*beatrice.barra@mail.polimi.com; phone+393384352786 
to accuracies up to $96 \%$ [10]. In addition, the ART 3.5 algorithm was also applied to a clinical dataset with a resolution of the dataset reduced of a factor 6 , leading to a reconstruction and segmentation of the vascular tree similar to the one obtained from the arteriography images. This result can be considered as a proof of concept for the functioning of ART 3.5D on real CTA data. Nonetheless, the strong limitations in resolution introduced in order to be able to process the real dataset do not allow the reconstruction of the capillaries.

This work aims at the introduction of the capillary structures in computational simulations of a vascular tree. The objective is to prove that also vascular structure with possibly different dynamic behavior can be reconstructed and classified. The presence of a wide temporal gap in the contrast transit dynamic due to the capillary phase can represent a facilitation for arteries and veins distinction. The modeling of this gap was not included in the previous study [10]. From the observation of clinical DSA-CBCT datasets it is easy to infer that the injected contrast medium invades the arteries very quickly, it is rapidly washed in through the arteries, it fades expanding in the huge network of unresolved capillaries, and next it reappears in veins for the final rapid wash-out. The identification of capillary structures then, can help, together with spatial continuity based regularization methods, to obtain a sharper arteries and veins separation. Moreover, while introducing the capillary network, a more complex contrast transit dynamics was considered.

\section{METHODS}

The ART 3.5D method has already been explained in our previous publication [10], and will be here summarized. In ART 3.5D the Algebraic Reconstruction Technique (ART) was extended dynamically, in order to account for dynamic changes of voxel intensity values in time, according to the progression of contrast medium. Voxel-wise dynamic variations were described by a Time Intensity Curve (TIC). TICs were modeled by the linear combination of a set of temporal basis functions in order to capture, by a minimal parameter set, regular wash-in wash-out features and approximate the timing sufficient to separate arteries and veins. Specifically, the general Linear Time Invariant (LTI) system for static image reconstruction,

$$
p=W \mu
$$

where $W$ is the system matrix, $\mu$ is the unknowns vector and $p$ is the measures vector, is transformed in a Linear Time Varian (LTV) system such as

$$
p_{d}=W_{d} \mu_{d}
$$

where the new Dynamic System Matrix $W_{d}$ includes time variant terms that depend on the basis functions chosen for the TIC modelling. The unknowns vector $\mu_{d}$ contains the new set of unknowns, which are the basis functions coefficients. Such coefficients allow to reconstruct a TIC for each voxel through their multiplication to the basis functions. The projection vector $p_{d}$ contains the projections replicated for all the time instants considered in the dynamic system. Once the coefficients were computed by solving this LTV system the TIC were reconstructed and a classification strategy based on the Area Under the Curve (AUC) of the TICs was applied. In particular, since arterial TICs have an AUC in the first half of the curve much greater that in the second half on the curve, while the venous TICs show a smaller AUC in the first half of the curve, the arterial Area Under the Curve $\left(\mathrm{AUC}_{\mathrm{A}}\right)$ was computed as the integral of the voxel's TIC up to the believed end of arterial phase and compared to an arterial threshold $\left(\mathrm{Th}_{\mathrm{A}}\right)$, to be exceeded for arterial classification. Within this work, since capillary structures were introduced in the vessel tree phantom, the AUC criterion was accordingly modified, meaning that the arterial and the venous Area Under the Curve $\left(A U C_{A}\right.$ and $\left.A U C_{V}\right)$ were computed respectively as the integrals of the voxel's TIC up to and from the believed end of arterial phase. If the ratio between the $\mathrm{AUC}_{\mathrm{A}}$ and the $\mathrm{AUC}_{\mathrm{v}}$ was greater than 1.5, the voxel was classified as artery, if smaller than 1.5, as a vein, otherwise as a capillary. A control of the value of the TIC peak was implemented as a regularization on the classification strategy, in order to avoid misclassification between capillaries and arterioles and venules.

In order to test the algorithm in presence of capillaries a phantom of the vascular tree including arteries, capillaries and veins was simulated. The vessel tree, represented in Figure 1, is composed of an artery that branches in three arterioles each on them branching in two capillaries, rejoining in three venules and finally in a vein. The whole image dimension in voxel is $50 \times 50 \times 50$ and the dimension of the isotropic voxels is $1 \mathrm{~cm}^{3}$. The diameters of the vessels are listed in Table 1 : they were set in order to have a vessel tree fitting in the image space, but and with relative dimensions reflecting real proportions. The experimental observations we rely on for the dynamic simulation are inferred from a CBCT dataset acquired with an acquisition protocol used at the Niguarda Hospital of Milan from the neurosurgeon Francesco Cardinale. In such clinical protocol, $12 \mathrm{cc}$ of iodate contrast medium are inserted in 1 second. Data on vessels diameters were taken from the same angiography images. The patient had written informed consent and the dataset was anonymized. 


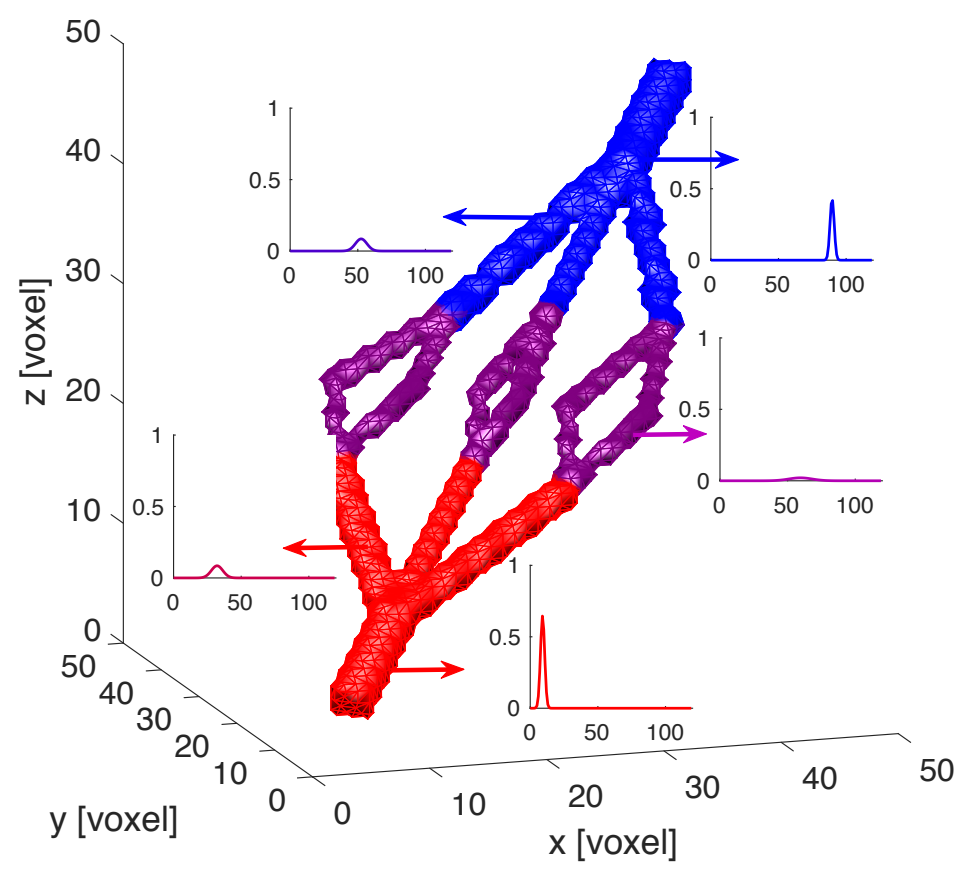

Figure 1. Simulated vascular tree comprising arteries (red), capillaries (purple) and veins (blue). For each type of vessel a qualitative representation of the simulated contrast curve is reported aside. On the $\mathrm{x}$ and $\mathrm{y}$ axis of each curve are reported, respectively, the number of samples and the normalized intensity value.

Table 1. Diameters set in simulations for different type of vessels.

\begin{tabular}{|c|c|c|c|}
\hline & ARTERIES/VEINS & ARTERIOLES/VENULES & CAPILLARIES \\
\hline DIAMETER & $3 \mathrm{~cm}$ & $1.8 \mathrm{~cm}$ & $0.5 \mathrm{~cm}$ \\
\hline
\end{tabular}

The fluid_dynamics in the whole vessel tree was mathematically computed using a 0D modeling approach. This model is well-known as lumped-parameter model and it exploits the analogy between electrical circuits and fluid flow. In fact this approach associates the flow and the driving pressure drop in the vessel segment to the current and the voltage respectively [11].

Following the electrical analogy for fluid dynamic, according to Ohm's law of hydrodynamics the pressure drop $\Delta \mathrm{P}$ $[\mathrm{mmHg}]$ across the branch can be defined as follows [12-13]:

$$
\Delta P_{i}=R_{i} Q_{i}
$$

where $R_{i}$ is the fluid resistance and $Q[\mathrm{cc} / \mathrm{s}]$ the flow rate in the branch.

Following Poiseuille's law [13], the fluid resistance was computed as:

$$
R_{i}=\frac{8 \pi \mu l}{S^{2}}
$$

Where $\mu[\mathrm{cP}]$ is the viscosity of the fluid, $l[\mathrm{~cm}]$ is the length of the vessel and $S\left[\mathrm{~cm}^{\wedge} 2\right]$ is the cross-section.

After the model is defined, specific boundary conditions have been imposed. In particular, the pressure at the inlet and outlet of the circuit has been defined as 35 and $10 \mathrm{mmHg}$ respectively, i.e. as the hydrostatic pressure encountered at the 
end of the arterioles and at the entrance of the venules. Once the flow rate was obtained in each branch, the concentration curve (CC) was computed as a modulation of a basis function over the specific flow value in the branch. The basis function exploited for the contrast concentration modeling (Eq. 5) is a Gaussian curve and therefore includes a rise phase and a drop phase, simulating then a bolus injection.

$$
C C(t)=\frac{1}{\sigma \sqrt{2 \pi}} \frac{e^{-(t-\mu)^{2}}}{2 \sigma^{2}}
$$

In Equation 5, the term $\mu$ represents the temporal position of the peak of the curve, therefore in our simulation, it corresponds to the value of the delay of the contrast transit in the voxel; the term $\sigma$ is the standard deviation of the gaussian distribution, and sets the width of the curve, being correlated, in our simulations, to the inverse of the velocity of the contrast medium flow in the voxel.

Based on this curve, the time intensity curve $(T I C)$ for the $\mathrm{i}^{\text {th }}$ voxel is computed as:

$$
T I C_{i}=\frac{Q_{i}}{Q_{0}} C C
$$

where $Q_{i}$ is the flow rate in the $\mathrm{i}^{\text {th }}$ voxel, and $Q_{0}$ is the flow rate at the vessel tree entrance, in the injection point. The obtained TICs for each type of vessel are shown in Figure 2.

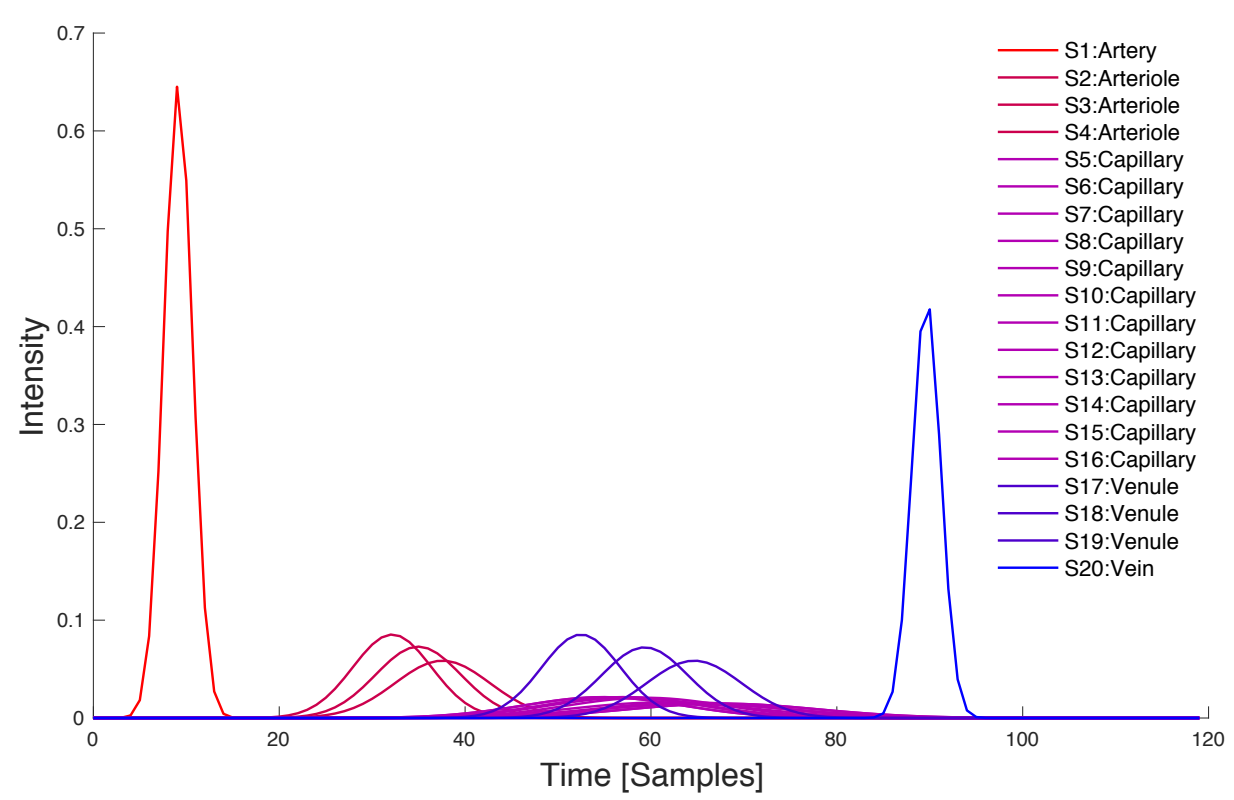

Figure 2. TICs simulated for the vascular tree. A TIC for each type of vessel is represented: arteries (red), arterioles (dark red) capillaries (purple), venules (dark blue) and veins (blue).

For the evaluation of the classification, the simulated vascular tree was used as a ground truth. divided in three masks for the arteries $\left(\mathrm{S}_{\mathrm{A}}\right)$, for the veins $\left(\mathrm{S}_{\mathrm{V}}\right)$ and for the capillaries $\left(\mathrm{S}_{\mathrm{C}}\right)$. The contingency tables for both arteries and veins classification were computed, together with sensitivity, specificity and accuracy values. In addition, indexes for the evaluation of cross-misclassification of arteries and veins were computed, and are defined as:

- The arteries to veins misclassification index AV

- The veins to arteries misclassification index VA

$$
A V=\frac{\left(C_{A}-S_{A}\right) \cap S_{V}}{S_{A}}
$$




$$
V A=\frac{\left(C_{V}-S_{V}\right) \cap S_{A}}{S_{V}}
$$

where $\mathrm{C}_{\mathrm{A}}$ are the classified arteries, $\mathrm{S}_{\mathrm{A}}$ are the simulated arteries, $\mathrm{C}_{\mathrm{V}}$ are the classified veins and $\mathrm{S}_{\mathrm{V}}$ are the simulated veins.

The simulated data were used to obtain simulated projection data, which were successively exploited to test the reconstruction algorithm and the following classification strategy.

\section{RESULTS}

The classification results for arteries, veins and capillaries are reported in Table 2, 3 and 4 . In addition, the misclassification indexed between arteries and veins (see Section 2) are reported in Table 5 and the accuracy, sensitivity and specificity values for both arteries and veins classifications are reported in Table 6 . The classification showed good results, with values between $60 \%$ and $90 \%$ of correctly classified arteries, capillaries and veins. Nevertheless, the classification performance for arteries lowered with respect to previous results. In fact, thought that there was not misclassification between arteries and veins, the classification of arteries suffered from misclassification with capillaries. Nonetheless, the cross-misclassification with capillaries can be addressed in the case on real dataset processing, pursuing an a-priori analysis of the dynamic characteristics of the contrast medium dynamics for the design of a spatial/temporal regularization technique.

Table 2. Contingency table for arteries classification.

\begin{tabular}{|c|c|c|}
\hline ARTERIES & POSITIVE & NEGATIVE \\
\hline POSITIVE & $60.59 \%$ & $0 \%$ \\
\hline NEGATIVE & $39.41 \%$ & $100 \%$ \\
\hline
\end{tabular}

Table 3. Contingency table for veins classification

\begin{tabular}{|c|c|c|}
\hline VEINS & POSITIVE & NEGATIVE \\
\hline POSITIVE & $94.93 \%$ & $0.02 \%$ \\
\hline NEGATIVE & $5.07 \%$ & $99.98 \%$ \\
\hline
\end{tabular}

Table 4. Contingency table for capillaries classification

\begin{tabular}{|c|c|c|}
\hline CAPILLARIES & POSITIVE & NEGATIVE \\
\hline POSITIVE & $94.43 \%$ & $0.15 \%$ \\
\hline NEGATIVE & $5.57 \%$ & $99.85 \%$ \\
\hline
\end{tabular}

Table 5. Misclassification values of arteries and veins among themselves.

\begin{tabular}{|c|c|c|}
\hline $\begin{array}{c}\text { MISCLASSIFICATION } \\
\mathrm{A} / \mathrm{V}\end{array}$ & AV & VA \\
\hline & $5.15 \%$ & $0 \%$ \\
\hline
\end{tabular}

Table 6. Accuracy, sensitivity and specificity values for arteries and veins classification.

\begin{tabular}{|c|c|c|c|}
\hline & ACCURACY & SENSITIVITY & SPECIFICITY \\
\hline ARTERIES & $80.30 \%$ & $100 \%$ & $71.73 \%$ \\
\hline VEINS & $97.45 \%$ & $99.98 \%$ & $95.17 \%$ \\
\hline CAPILLARIES & $97.14 \%$ & $99.84 \%$ & $94.71 \%$ \\
\hline
\end{tabular}

\section{CONCLUSIONS}

This work follows the study on the ART 3.5D suitability for arteries and veins separate classification from CE-CBCT datasets [10]. In particular, here the simulation of the capillary structures and temporal dynamics is introduced as an additional feature to the simulated vascular tree. In fact, as already pointed out in the previous article [10], the presence of a capillary temporal phase on real datasets can result in a remarkable facilitation since it causes a wide temporal gap between the arterial wash-in phase and the venous wash-out phase. Here the obtained results show a comparable performance with respect to the previously published results [10], confirming the hypothesis that a different dynamic in 
different types of vessels would not be a problematic feature in real dataset processing. In addition, the crossmisclassification among arteries and veins lowered, confirming that the capillary phase can serve as a facilitation for arteries and veins distinction. Differently, the misclassification of arteries and veins with capillaries is higher, leading to lower rates of correct classification for both the types of vessels. Nonetheless, in order to correct this classification error, several spatial and temporal regularization techniques can be introduced in case of real dataset. We therefore believe that the presence of capillary structures can however be addressed and exploited for better classification results in case of clinical CBCT datasets. Future work will mainly be focused on the introduction of spatial and temporal regularization techniques and the introduction of an appropriate computational framework relying on a more powerful hardware and exploiting parallel computation techniques in order to make the algorithm suitable for real images processing of CECBCT datasets.

\section{ACKNOWLEDGEMENTS}

The present work has been supported and advised by Medtronic, Minneapolis, USA. Dr. Cardinale reports receiving consulting fees from Renishaw-Mayfield since 2010.

\section{REFERENCES}

1. D. Babin, A. Pizurica, J. De Vylder, E. Vansteenkiste, and W. Philips, "Brain blood vessel segmentation using line-shaped profiles" Physics in medicine and biology 58(22), 8041 (2013).

2. M. G. Safain, J. P. Rahal, A. Raval, M. J. Rivard, J. E. Mignano, J. K. Wu, and A. M. Malek, "Use of conebeam computed tomography angiography in planning for gamma knife radiosurgery for arteriovenous malformations: A case series and early report", Neurosurgery, 74(6), 682- 696 (2014).

3. M. Nowell, R. Rodionov, G. Zombori, R. Sparks, G. Winston, J. Kinghorn, B. Diehl, T. Wehner, A. Miserocchi, A. W. McEvoy, et al., "Utility of 3d multimodality imaging in the implantation of intracranial electrodes in epilepsy", Epilepsia 56(3), 403-413 (2015).

4. F. Cardinale, M. Cossu, L. Castana, G. Casaceli, M. P. Schiariti, A. Miserocchi, D. Fuschillo, A. Moscato, C. Caborni, G. Arnulfo, et al., "Stereoelectroencephalography: surgical methodology, safety, and stereotactic application accuracy in 500 procedures", Neurosurgery 72(3), 353-366 (2013).

5. F. Cardinale, "Talairach methodology in the era of $3 \mathrm{~d}$ multimodal imaging:the song remains the same, but catchier, and therefore more helpful for clinical decision making and surgical planning in epilepsy surgery" Epilepsia 56(6), 976-977 (2015).

6. F.Cardinale, G.Pero, L.Quilici, M.Piano, P.Colombo, A.Moscato, L.Castana, G.Casaceli, D. Fuschillo, L. Gennari, et al., "Cerebral angiography for multimodal surgical planning in epilepsy surgery: description of a new three-dimensional technique and literature review", World neurosurgery 84(2), 358-367 (2015).

7. F. Cardinale, A. Miserocchi, A. Moscato, M. Cossu, L. Castana, M. Schiariti, F. Gozzo, G. Pero, L. Quilici, A. Citterio, et al., "Talairach methodology in the multimodal imaging and robotics era", Stereotaxy and epilepsy neurosurgery. John Libbey Eurotext, London , 245-272 (2012).

8. T. Lei et al., "Artery-vein separation via MRA-an image processing approach," IEEE Trans. Med. Imaging 20(8), 689-703 (2001).

9. H. Laue et al., "Automated artery and vein detection in 4D-CT data with an unsupervised classification algorithm of the time intensity curves," Proc. SPIE 8669, 86691W (2013).

10. Barra B., De Momi E., Ferrigno G., Pero G., Cardinale F., Baselli G., "ART 3.5D: an algorithm to label arteries and veins from three-dimensional angiography”, J. Med. Imag. 3(4), 044002 (2016), doi: 10.1117/1.JMI.3.4.044002.

11. I. Kokalari, T. Karaja, M. Guerrisi, "Review on lumped parameter method for modeling the blood flow in systemic arteries", Journal of Biomedical Science and Engineering, 6(1), 92 (2013).

12. S. Lorthois, F. Cassot, F. Lauwers, "Simulation study of brain blood flow regulation by intra-cortical arterioles in an anatomically accurate large human vascular network: Part I: Methodology and baseline flow", NeuroImage, 54(2), 1031-1042, (2011).

13. E. Gabryś, M. Rybaczuk, A. Kędzia, "Blood flow simulation through fractal models of circulatory system", Chaos, Solitons \& Fractals, 27(1), 1-7, (2006). 